\title{
MECHANICAL PROPERTIES OF 3D-STRUCTURE COMPOSITES BASED ON WARP-KNITTED SPACER FABRICS
}

\author{
Si Chen ${ }^{1,2}$, Hai-ru Long ${ }^{1,2}$, Ying-hao Liü, Feng-chao $\mathrm{Hu}^{4}$ \\ ${ }^{1}$ College of Textiles, Donghua University, Shanghai 201620, China \\ ${ }^{2}$ Key Laboratory of Textile Science \&Technology, Ministry of Education, China \\ ${ }^{3}$ R\&D building, BASF PuDong Site, Shanghai 200100, China \\ ${ }^{4}$ BAPS, BASF PuDong Site, Shanghai 200100, China \\ E-mail: ansn9119@126.com
}

\begin{abstract}
:
In this paper, the mechanical properties (compression and impact behaviours) of three-dimension structure (3D-structure) composites based on warp-knitted spacer fabrics have been thoroughly investigated. In order to discuss the effect of fabric structural parameters on the mechanical performance of composites, six different types of warp-knitted spacer fabrics having different structural parameters (such as outer layer structure, diameter of spacer yarn, spacer yarn inclination angle and thickness) were involved for comparison study. The 3D-structure composites were fabricated based on a flexible polyurethane foam. The produced composites were characterised for compression and impact properties. The findings obtained indicate that the fabric structural parameters have strong influence on the compression and impact responses of 3D-structure composites. Additionally, the impact test carried out on the 3D-structure composites shows that the impact loads do not affect the integrity of composite structure. All the results reveal that the product exhibits promising mechanical performance and its service life can be sustained.
\end{abstract}

\section{Keywords:}

3D-strucutre composites, warp-knitted spacer fabrics, mechanical properties

\section{Introduction}

Light-weight construction, from the economical points of view, is of particular importance in the range of modern industrial systems [1]. Composites with integrated 3D structure offer the great potential to be used in engineering applications, since they combine low weight with high mechanical properties and their delamination resistant abilities are also preferable. The 3D-structure composites are usually made from spacer fabrics, consisting of two separate faces connected by spacer yarns. The composites made with these 3D-structure fabrics have the following advantages: (1) composites can be fabricated in one single step instead of complicated manufacturing process; (2) these two faces of fabrics are bound by spacer yarns, thus the face-core delamination is impossible; (3) the hollow core area can be filled with a variety of materials, depending on different end-use requirements. In these circumstances, the integrated 3D-structure composites are widely used for automobiles, vehicles, marine and so on.

Warp-knitted spacer fabrics having specific 3D structure are recently used for the fabrication of 3D-structure composites [2]. As a reinforcement material, warp-knitted spacer fabrics have much better moisture transmission features [3], better resilience properties [4] and higher air permeability [5]. Moreover, composites reinforced by warp-knitted spacer fabrics exhibit superior mechanical performance compared to other textilebased composites [6-9]. Additionally, the mechanical properties of warp-knitted spacer fabric composites can also be tailored to meet the specific applications by simply varying the fabric structural parameters.
Studies on mechanical behaviours of composites based on 3D warp-knitted fabrics and unsaturated resin have been investigated recently by Velosa et al. [2] Some effect factors, that is, cross-thread density, fineness of yarns and structure of face of fabrics, as well as thickness of fabrics, on the mechanical behaviours have been discussed in this study. Vuure et al. [10] developed a unit-cell model of core properties of composite panels based on spacer fabrics, and the finite element method calculations of the compression responses of these novel composites have been performed. Thermoplastic composites based on flat warp-knitted 3D multilayer spacer fabrics have been introduced by Abounaim [6-9]. It can be concluded from their studies that the mechanical responses of these composites are significantly influenced by the arrangement of reinforced yarns and the integration of reinforced yarns with biaxial inlays are found to be the optimum method. However, none of researchers has concentrated on the flexible composites based on spacer fabrics. The 3D-structure composites involved in this study possess a flexible structure, which is quite different from the composites made with stiff surfaces as shown in literatures [3-10]. The novel integrated 3D-structure composites are expected to be used for cushioning applications, such as the car seat, package materials, protection materials, and so on in order to reduce fractures after suffering compression or impact loads.

The present work reports the mechanical properties (compression and impact) of 3D-strucutre composites manufactured based on warp-knitted spacer fabrics. With an attempt to discuss the effect of fabric structural parameters on the mechanical properties of 
composites, a series of warp-knitted spacer fabrics with different parameters (such as outer layer structure, diameter of spacer yarn, spacer yarn inclination angle and thickness) were used to fabricate the composites. It is expected that a regular pattern for tailoring the composites with favourable mechanical behaviours could be found from this study.

\section{Experimental details}

\subsection{Samples}

\subsubsection{Warp-knitted spacer fabrics}

Six types of warp-knitted spacer fabrics (hereinafter referred to briefly as WSF) used in this work have been produced on a double-needle-bar Raschel warp knitting machine of E18 (Wuyang Co. Ltd., Jiangsu, China). The polyethylene terephthalate monofilament of 0.2 and $0.16 \mathrm{~mm}$ in diameter were used for spacer yarns, while 300D/96F PET multifilament were used as surface layer yarns. The surface structures of spacer fabrics are Chain+Inlay, Locknit and Hexagonal Mesh, as shown in Figure 1 (a)-(c). According to their shapes, the outer layer structures can be divided into two categories: the close structure without mesh (Chain+Inlay and Locknit) and open structure with mesh (Hexagonal Mesh). In addition, it can be revealed that Locknit is slightly closer than Chain+Inlay. Two different fabric thicknesses and spacer yarn inclination angle were also involved. All the samples were heat-set at $180^{\circ} \mathrm{C}$ for $3 \mathrm{~min}$. The resultant details of all samples are listed in Table1. Despite the same setting of warping machine, actual thickness of WSF1, WSF2, WSF3, WSF5 and WSF6 were slightly different, but they were all approximately $7.4 \mathrm{~mm}$; thus, it can be considered that the thickness of these fabrics were close to each other.

\subsubsection{Preparation of the composites}

The composites (hereinafter referred to briefly as PWSF) were produced by impregnating the warp-knitted spacer fabrics with a flexible polyurethane foam, consisting of a mix of $1.21 \mathrm{~g} / \mathrm{cm}^{3}$ isocyanate and $0.78 \mathrm{~g} / \mathrm{cm}^{3}$ polyol, in a $43.7 / 100$ isocyanatepolyol mixing ratio (by weight) (BASF, PuDong Site, Shanghai, China). The important properties of this polyurethane foam are

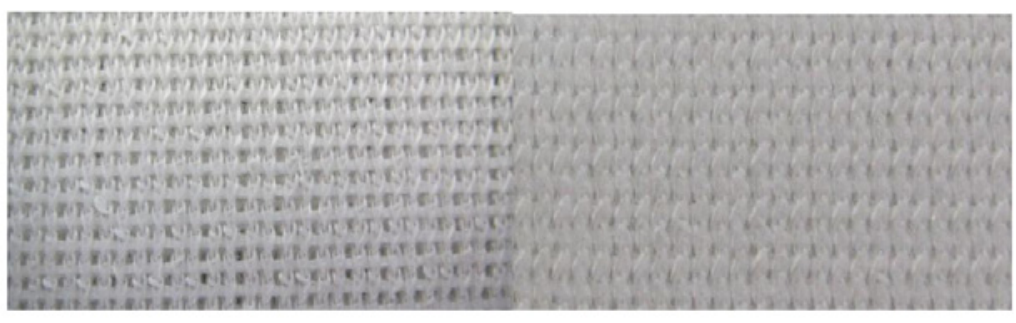

(a)

(b)

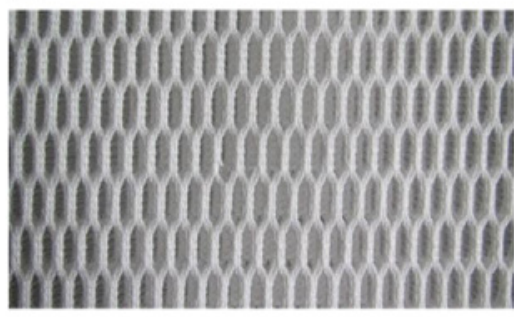

(c)

Figure 1. The surface layer structures (a) Chain+Inlay (b) Locknit (c) Hexagonal Mesh

Table 1. Structural parameters of warp-knitted spacer fabric

\begin{tabular}{|c|c|c|c|c|}
\hline Code & Thickness $\mathbf{( m m )}$ & $\begin{array}{c}\text { Surface layer } \\
\text { structure }\end{array}$ & $\begin{array}{c}\text { Diameter of spacer yarn } \\
\mathbf{( m m})\end{array}$ & $\begin{array}{c}\text { Spacer yarn inclination } \\
\text { angle (degree) }\end{array}$ \\
\hline WSF1 & 7.46 & C & 0.2 & 80 \\
\hline WSF2 & 7.44 & C & 0.16 & 65 \\
\hline WSF3 & 7.42 & C & 0.2 & 65 \\
\hline WSF4 & 5.96 & C & 0.2 & 80 \\
\hline WSF5 & 7.42 & H & 0.2 & 80 \\
\hline WSF6 & 7.44 & L & 0.2 & 80 \\
\hline
\end{tabular}

'C', 'H' and 'L' represent Chain+Inlay, Hexagonal Mesh and Locknit, respectively. Spacer yarn inclination angle is defined as the acute angle between the spacer yarn and fabric surface layers. 
listed in Table 2. Production of the composite samples was carried out in a mould, of which the top and bottom surfaces can be heated by a water bath heating system, as shown in Figure 2. The isocyanate-polyol mixing liquid was infused through the core of spacer fabrics in the warp direction. At the same time, the water temperature was maintained at $40^{\circ} \mathrm{C}$ during the infusion process that was carried out for $8 \mathrm{~min}$, in order to maintain the cured polyurethane foam. After foaming, all the specimens were placed for 24 hours at room temperature, until the polyurethane foam was stable. The six types of composites produced are listed in Table 3. The scanning electron microscope images of polyurethane foam and $x-y$ plane cross-section of composite are shown in Figure 3. The real view and schematic diagram of produced composites are shown in Figure 4.

\subsection{Distribution of polyurethane foam tests}

Table 2. Properties of polyurethane foam

\begin{tabular}{|c|c|}
\hline Property & Values \\
\hline Density $\left(\mathrm{g} / \mathrm{cm}^{3}\right)$ & 0.15 \\
\hline Tensile modulus (kpa) & 89.8 \\
\hline Elongation at break (\%) & 147.5 \\
\hline Compression strength (kpa) & 58.7 \\
\hline Compression modulus (kpa) & 75.2 \\
\hline
\end{tabular}

Since the distribution condition of polyurethane foam in composites could significantly influence the mechanical properties of composites, it is necessary to stress whether the polyurethane foam is well-distributed in the composites. Thus, a Compact X-RAY Density Profile Analyzer (IMAL DPX300LTE, Italy) was used to evaluate the distribution condition of polyurethane foam in composites. The working principle of this instrument is shown in Figure 5. The DPX300-LTE is a compact laboratory machine that is used to supply the density profile of particleboard, measured along the thickness. The samples may be of varying thickness and may be measured individually or in groups so as to highlight any particular differences in material distribution at various points on the same board (e.g. different distribution on the two sides of the same board). When scanning a given object, the X-RAY moved along the $\mathrm{x}$-axis. The interval of the $\mathrm{x}$-axis was $0.1 \mathrm{~mm}$.

\subsection{Compression tests}

The compression test was conducted on all the specimens using the Shanghai HL WDW Series material test instrument, and the test was done at $23^{\circ} \mathrm{C}$ and $65 \%$ relative humidity based on GB/T8168-2008 test standard. The size of compression platen was $\Phi=100 \mathrm{~mm}$, whereas all samples were circular in shape with a diameter of $60 \mathrm{~mm}$. The samples were pressed to deformation to $60 \%$ of the initial thickness at a load speed of 5 $\mathrm{mm} / \mathrm{min}$ and five repeats were carried out for each specimen. Each compression stress-strain curve was an average of the five experimental results.

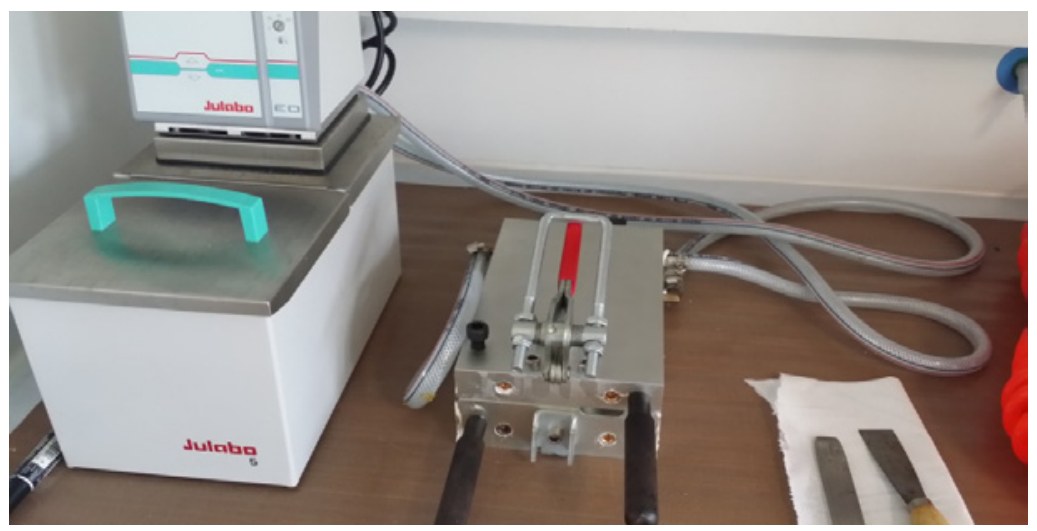

Figure 2. The instrument for polyurethane-based composites fabrication

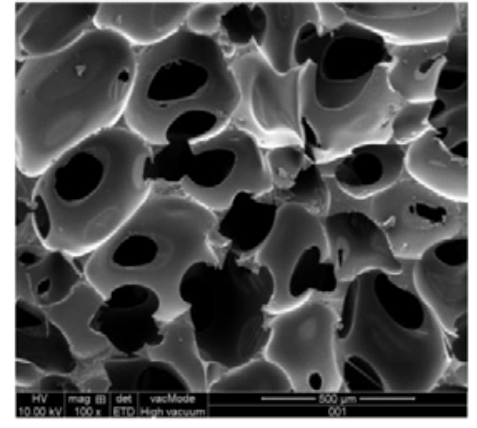

(a)

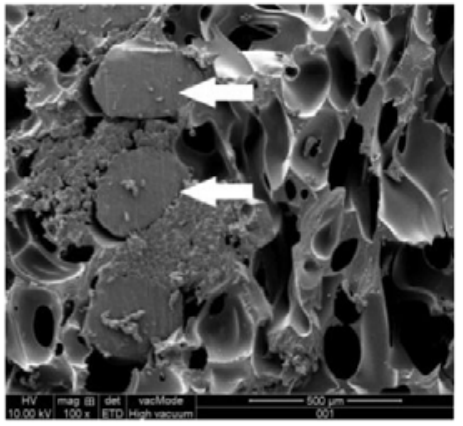

(b)

Figure 3. SEM images of polyurethane foam (a) and $x-y$ plane cross-section of composite (b) (the arrows in $b$ indicate the spacer yarns in composite) 


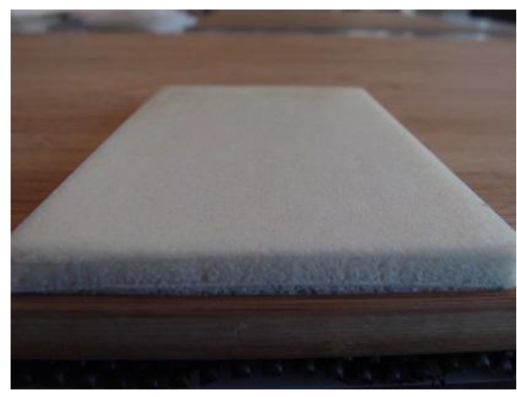

(a)

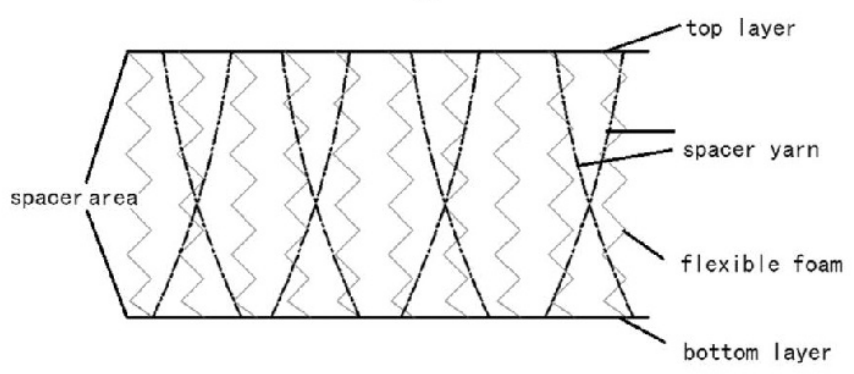

(b)

Figure 4. The real view (a) and schematic diagram (b) of PWSF

Table 3. Details of the composites

\begin{tabular}{|c|c|c|c|}
\hline Sample & $\begin{array}{c}\text { Thickness } \\
(\mathbf{m m})\end{array}$ & $\begin{array}{c}\text { Fibre volume } \\
\text { fraction } \\
\mathbf{( \% )}\end{array}$ & $\begin{array}{c}\text { Density } \\
\mathbf{( k g / \mathbf { m } ^ { 3 } )}\end{array}$ \\
\hline PWSF1 & 7.72 & 7.33 & 266.3 \\
\hline PWSF2 & 7.76 & 6.57 & 250.3 \\
\hline PWSF3 & 7.66 & 6.32 & 249.7 \\
\hline PWSF4 & 6.60 & 8.91 & 244.2 \\
\hline PWSF5 & 7.86 & 2.25 & 249.2 \\
\hline PWSF6 & 7.82 & 8.25 & 284.1 \\
\hline
\end{tabular}

\subsection{Impact tests}

Impact properties of the composites were evaluated using an Instron Dynatup 9250HV according to GB/T8171-2008 test standard, as shown in Figure 6. An impactor having a diameter of $25 \mathrm{~mm}$ with a flat tip was used to hit against the face of specimens (100'100 mm). The weight of the impactor was $6.017 \mathrm{~kg}$. Incident impact energy used for the work was 10 $\mathrm{J}$ and $15 \mathrm{~J}$, respectively. For each composite, three samples were tested and the ultimate result was the average of the three tests.

\section{Result and discussion}

\subsection{The distribution of polyurethane foam}

Investigation using the X-RAY Density Profile Analyzer gives an indirect measurement of the distribution of polyurethane foam in composite during the composite manufacturing process. This is due to the fact that the distribution of polyurethane foam

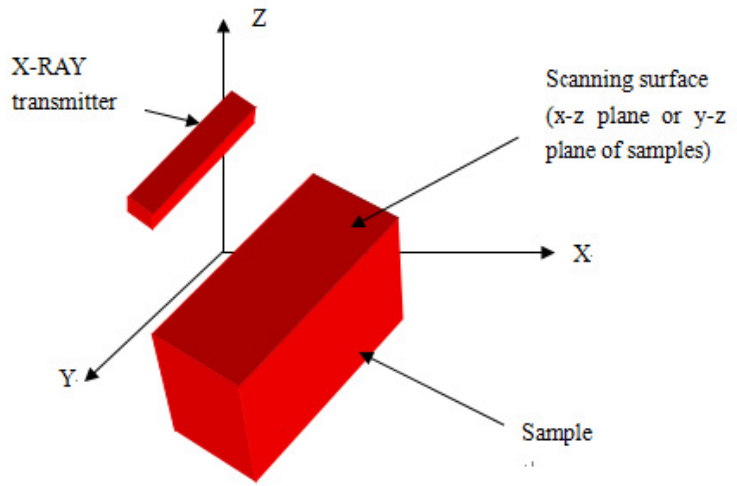

Figure 5. Working principle of X-RAY Density Profile Analyzer

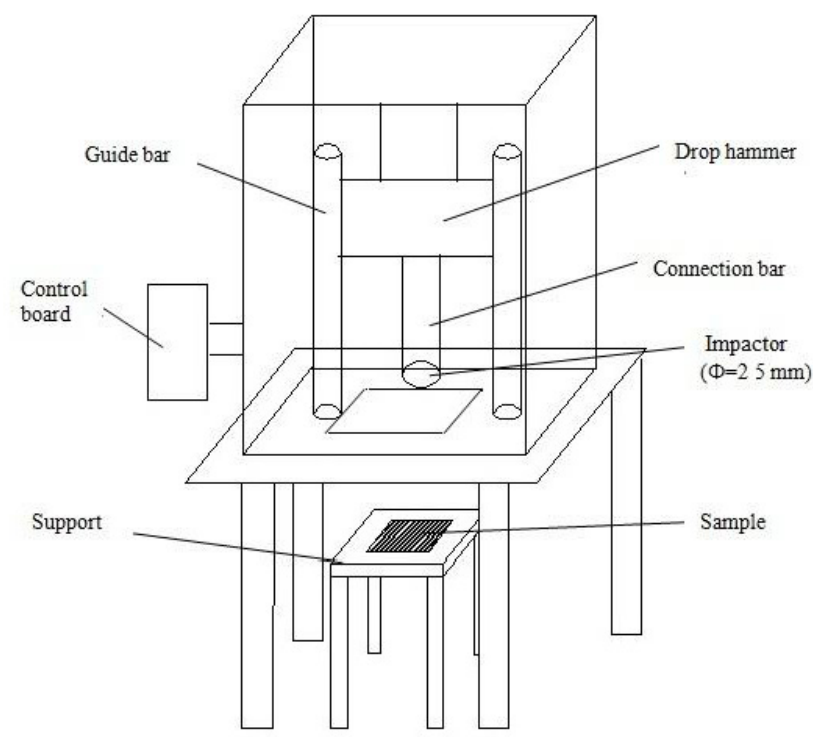

Figure 6. Impact instrument structure 
in composites is supposed to be even under the condition that the density values in the spacer area of composites are kept constant and the density values in top layers are close to that of bottom layers.

The density values of sample PWSF2, PWSF3 and PWSF6, which were chosen as the representative of six samples, are presented in Figure 7. In Figure 7, the beginning (the thickness value is about $0-1.5 \mathrm{~mm}$ ) and end (the thickness value is about $6.5-7.8 \mathrm{~mm}$ ) of the curves represent the density values in top and bottom layers of composites, respectively, whereas the middle of curves (the thickness value is about $1.5-6.5 \mathrm{~mm}$ ) reflects the density values in spacer area of composites. It can be seen that the density values in surface layers of composites are higher compared to the density values in the spacer area of composites. This phenomenon can be explained by the fact that the density values in outer layers of spacer fabrics are higher compared to the density values in spacer area, resulting in higher density values in the surface layers of composites. However, it is obviously observed that the density values in spacer area of composites are nearly constant along the direction of thickness, indicating that the distribution of polyurethane foam is uniform in the spacer area of composites. Additionally, the density values in top and bottom layers of composites are also very close to each other. All the results significantly demonstrate that the polyurethane foam exhibits a fairly well-distributed pattern in the composites.

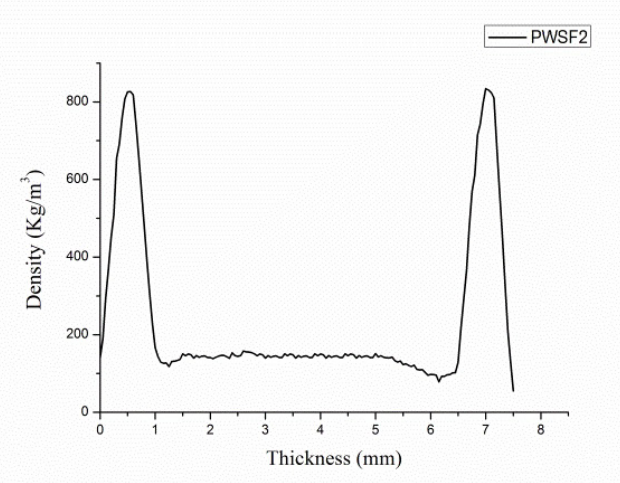

(a)

\subsection{Compression behaviours of composites}

A typical compression stress-strain curve of composite (PWSF1) is shown in Figure 8. It is obvious that a plateau region can be observed in the stress-strain curve. In the plateau region, a nearly constant stress is obtained. However, after this region, there is a rapid increase in the stress value due to densification of the entire composite. It is well known that from the initial region to the end of plateau region, the area under the stress-strain curve represents the energy absorbed by the composite within a fairly constant stress value. Thus, it can be concluded from Figure 8 that the composites possess good energy absorption capability, because a nearly constant stress value in plateau region is obtained until a large displacement, which is beyond half of the initial thickness and corresponding to a strain of almost $50 \%$. The specific properties are just the requirement for a good energy absorber. However, it is necessary to point out that after the plateau region, the energy absorbed by the composite is low, but the stress value is high. Therefore, for an energy-absorbing application, it is preferable that the composites should absorb all the energy before reaching the end of plateau stage.

With the aim of investigating the influence of fabric structural parameters on the compression behaviours, the stress-strain curves for all the six samples are shown in Figure 9. At the same time, the compression modulus $(\sigma)$ and energy absorbed

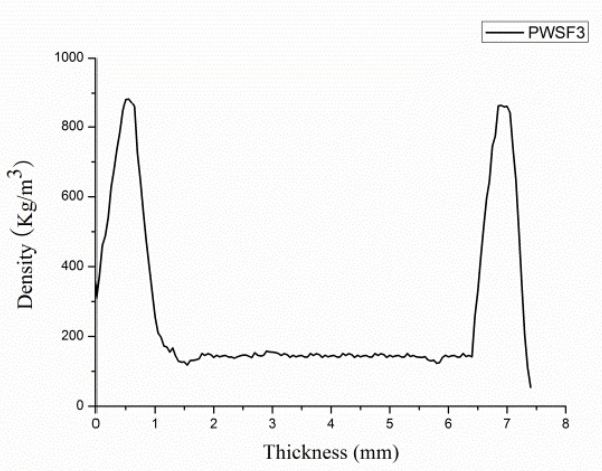

(b)

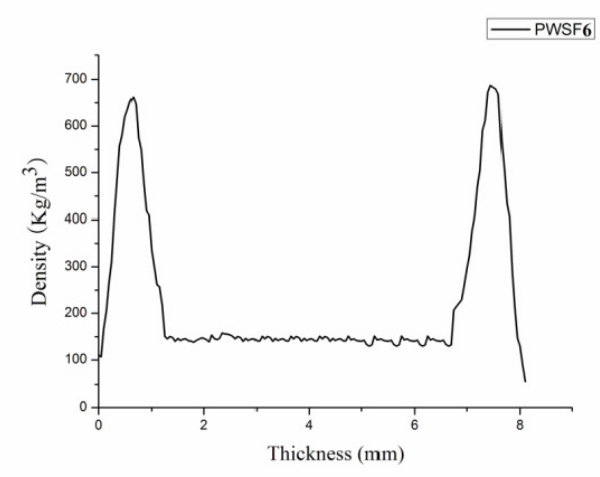

(c)

Figure 7. Density profile of composites 
$(U)$ during the compression process for all the samples are provided in Figure 10. It is evident that the fabric structural parameters have significant influence on the compression behaviours of composites.

PWSF1, PWSF5 and PWSF6 represent the composites with the same fineness and inclination angle of spacer yarn but the different outer layer structures. Furthermore, the thicknesses of these three samples are very close. If the performance of these three samples under compressive load is compared, it can be observed that the compression resistance capacities of composites decrease as the surface layer structure opens up. PWSF5 obtains the lowest compression resistance capacities among the three composites. It was mentioned earlier that PWSF5 has surfaces with open structures and lowest fibre content, resulting in the weakest face strength and lowest compression resistance abilities. On the contrary, PWSF6 produced the strongest face because of its compact face structure because of considerably high fibre content. Therefore, the highest compression resistance capacities stress was obtained in case of PWSF6. On the other hand, PWSF5 exhibits the shortest plateau region, leading to the lowest energy-absorbing performance. In contrast, the plateau region for PWSF6 is slightly longer than that of PWSF1 and PWSF5, indicating that PWSF6 can absorb more total energy compared to the corresponding samples, as shown in Figure 10.

PWSF2 and PWSF3 represent the composites with the same inclination angle of spacer yarn, but with different spacer yarns' diameters, which are 0.16 and $0.2 \mathrm{~mm}$, respectively. These two specimens also have the similar thickness and outer layer density. From the comparison result shown in Figure 9, it can be seen that composite PWSF3 was found to be better with respect to compression resistance capacities performance. This is attributed to the coarser spacer yarn used in this composite, leading to higher load carrying capability. Also, the plateau region for PWSF3 is longer than that of PWSF2, which indicates that composite made from coarser spacer yarn can absorb more energy, compared to the composite with finer spacer yarn.

PWSF1 and PWSF3 represent the composites with different inclination angle of spacer yarn. However, the other parameters of these two samples are very close. Referring to Figure 9, it is evident that the composite with larger inclination angle of spacer yarn exhibits higher compression resistance capacities performance. This phenomenon shows great agreement with the previous studies [11-13]. The larger the spacer yarn angle, the better the compression resistance capacities performance. Additionally, the plateau region is shorter for composite made from smaller angle of spacer yarn than that of composite made with larger angle of spacer yarn, leading to an increase of energy absorbed for composite with larger spacer yarn inclination angle, as shown in Figure 10.

PWSF1 and PWSF4 represent the composites with same outer layer structure and fineness of spacer yarn, but different thickness, which are 8.74 and $6.68 \mathrm{~mm}$. It can be seen from data provided in Figure 9 that the thinner composite exhibits

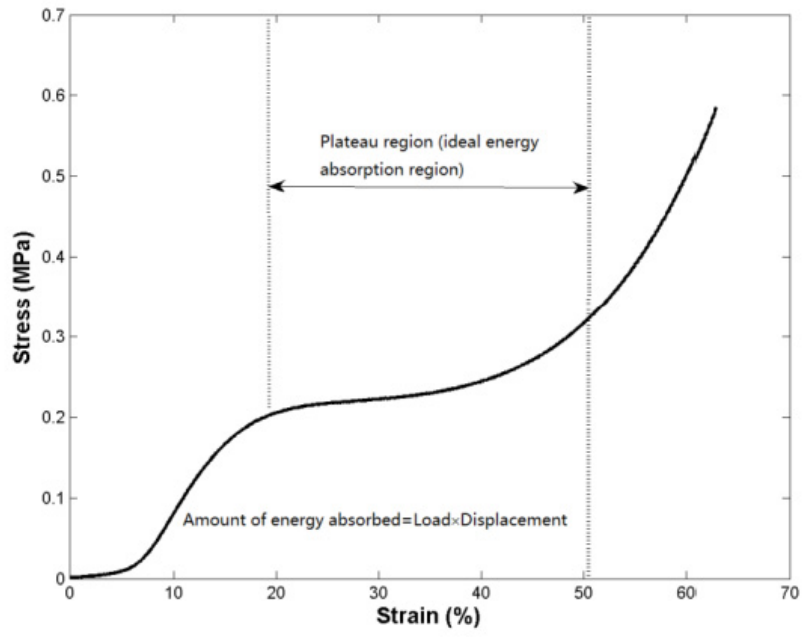

Figure 8. Typical compression stress-stain curve of composite

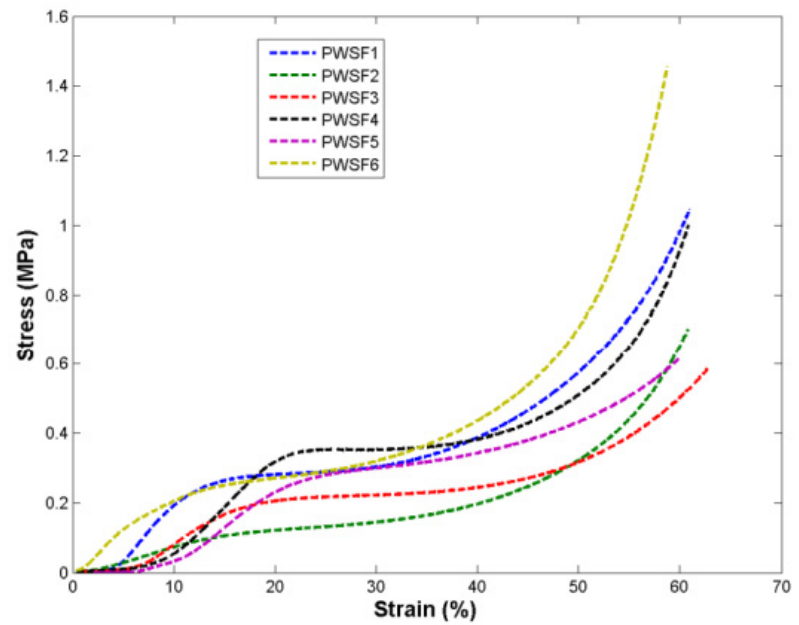

Figure 9. Compression stress-strain curves for all samples

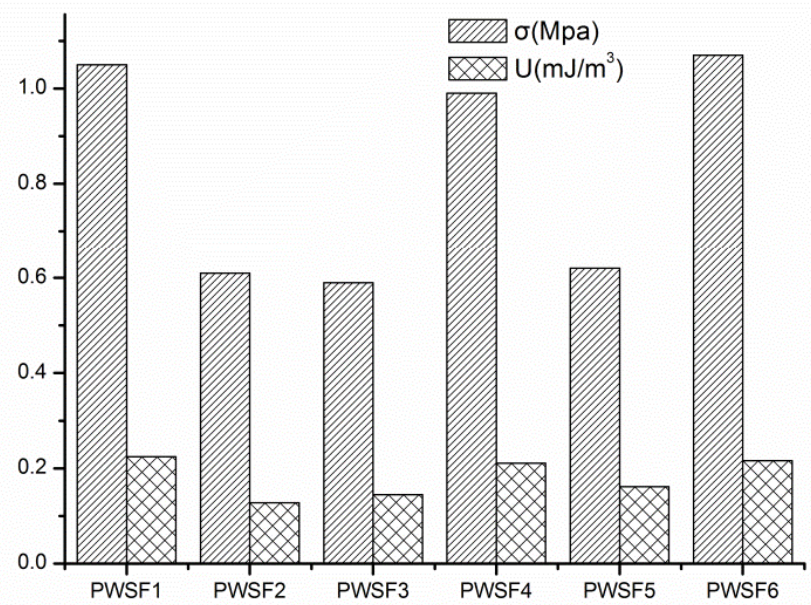

Figure 10. Compression modulus and energy absorbed for all samples

lower compression resistance abilities compared to the thicker composite at lower strain less than about $20 \%$. However, after this strain level, their compression behaviours reverse. The compression responses of PWSF4 are the inverse of PWSF1. 
In these circumstances, a conclusion can be drawn that the composites made by different thickness have their own ranges of applications. It is inappropriate to simply compare the compression and energy absorption capacities of composite made with different thickness.

\subsection{Impact behaviours of composites}

To analyze the typical impact curve of composites during impact process, PWSF3 is taken as a representative of six samples: its force-time and force-displacement curves are listed in Figure 11 (a) and (b), respectively.

Referring to Figure 11, it can be found that the curves are bound to change with the changes of incident energy values. The maximum displacement (at peak force) increases as the contact duration time decreases with the increase of impact energy. Force drops significantly and displacement declines to some position in the unloading stage. It can also be observed that the force values fluctuate around zero and finally increase to their values. It is attributed to the fact that the vibrations of the machine existed in actual. Furthermore, the vibrations between the impactor and the impact preventer also existed. Since they are both rigid objects, the impactor cannot stop immediately after rebounding and being held by the impact preventer. In contrast, it will stop gradually with the decrease of vibrations. Similarly, it should be stressed that the loading stage is an important factor for energy-absorbing behaviours, as the force values are nearly constant, whereas the displacement increases in this stage. The absorbed energy can be illustrated by the area under the impact force-displacement curves, as shown in Figure 11 (b).

The impact energy applied by the impact system is mainly withstood by the changes of displacement as a result of the bending of spacer yarns. Figure 12 (a)-(c) show the status of original spacer yarns and impacted spacer yarns under lower $(10 \mathrm{~J})$ and higher $(15 \mathrm{~J})$ impact energy, respectively (the original spacer yarns bend slightly due to the knitting technology). A continual increase of force for composites subjected to impact energy is the result of increasing displacement and bending of spacer yarns. Furthermore, the degree of bending of spacer yarns is relative to the incident energy. The higher the incident energy, the more the spacer yarns bend. However, it can be clearly observed that the spacer yarns are not damaged when subjected impact energy $(10 \mathrm{~J}$ and $15 \mathrm{~J}$ ). The specific behaviours of composites are due to fact that the polyurethane foam in the spacer area is equivalent to the foam-core of $3 D$ composites, indicating that the foam-core and $3 \mathrm{D}$ structure could support each other. While the foam-core constitutes a vertical support for the 3D structure, the integrated 3D structure forms a structure that prevents the foam-core from breaking and keeps the whole structure integrity.

The energy-time curves of PWSF3 under impact energy of $10 \mathrm{~J}$ and $15 \mathrm{~J}$ are shown in Figure 13. The impact energy given by the impactor can be divided into two parts: one is the rebounded energy, which is stored by samples or transferred back to the impactor. The other is the absorbed energy, which is absorbed by samples and impact system in vibration, heat,

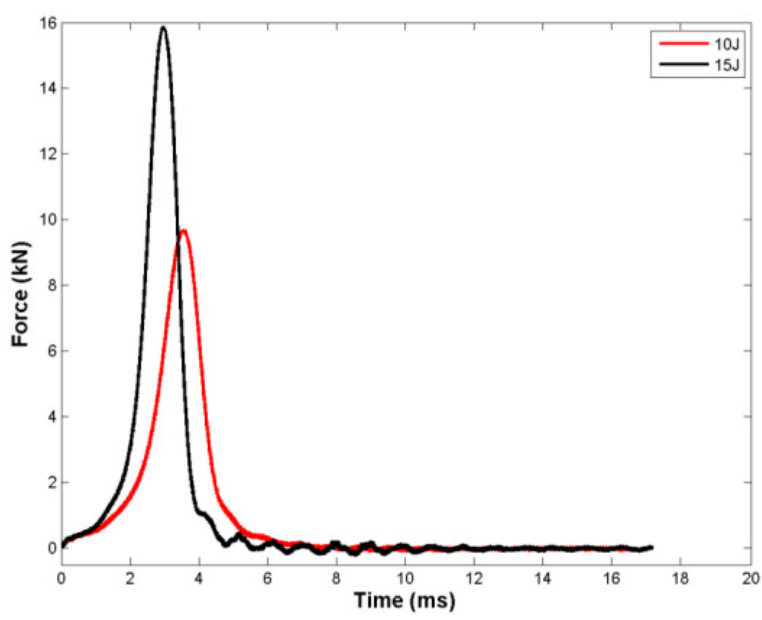

(a)

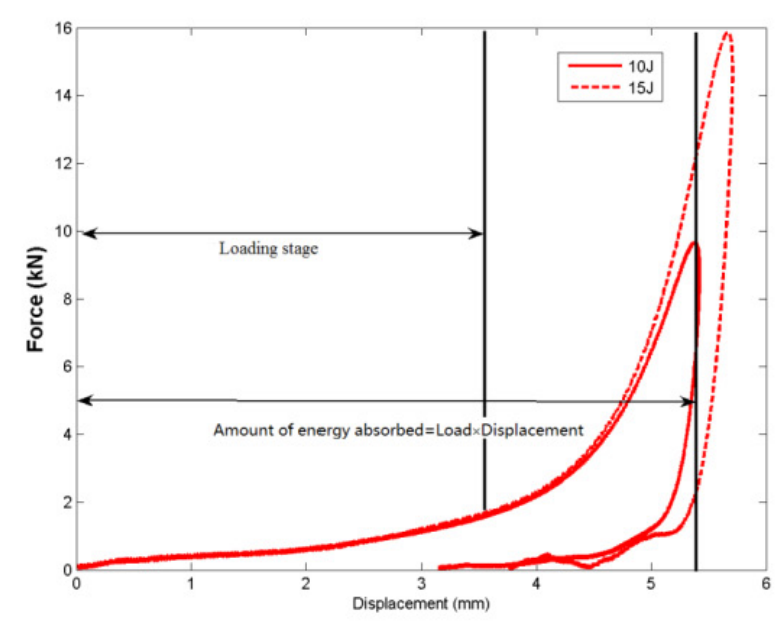

(b)

Figure 11. Typical force-time/displacement curve

supports and so on. Thus, the total absorbed energy can be given as following [16]:

$$
\mathrm{E}_{\text {total }}=\mathrm{E}_{\mathrm{r}}+\mathrm{E}_{\mathrm{a}} \wedge^{*} \text { MERGEFORMAT }
$$

Where $E_{\text {total }}$ is the total impact energy, $E_{\mathrm{r}}$ is rebounded energy, and $E_{\mathrm{a}}$ is the absorbed energy.

It should be stressed that the amount of energy absorbed by impact system is very small, thus, they can be ignored during the impact process. As a result, the total energy is the sum of rebounded energy and absorbed energy by samples. In particular, the amount of energy absorbed increases with the increasing of impact energy, and the undamaged material still can absorb a substantial amount of the impact energy for all energy levels.

With an attempt to discuss the influence of fabric structural parameters on the impact behaviours, the impact curves for all samples subjected different incident energy are shown in Figure 14 (a) and (b). For different composites conducted to the same impact energy, an ideal energy-absorber is considered to absorb as much energy as possible, which means that the 


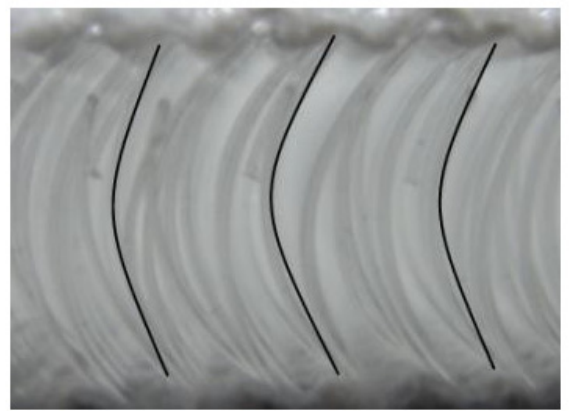

(a)

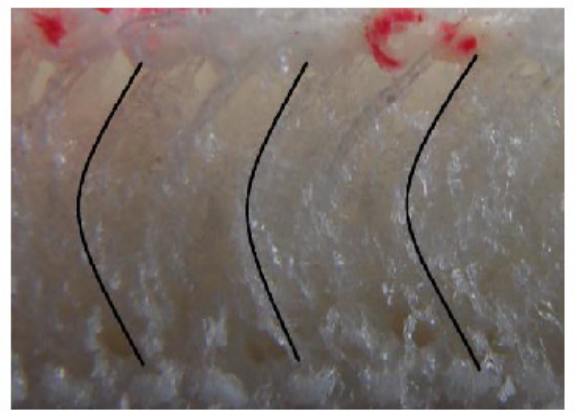

(b)

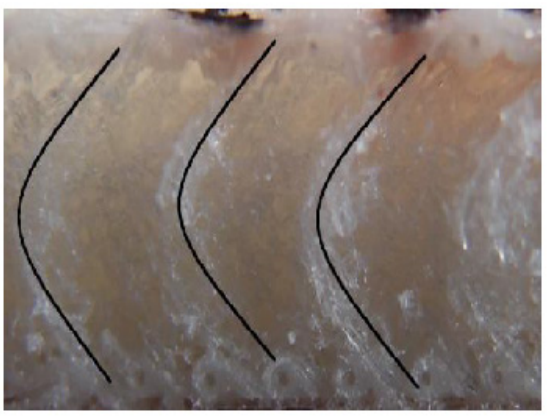

(c)

Figure 12. Status of spacer yarns (a) original (b) $10 \mathrm{~J}$ impact energy (c) $15 \mathrm{~J}$ impact energy

loading stage should be as long as possible [14-15]. The peak force and absorbed energy for all the samples are provided in Figure $15(\mathrm{a})$ and (b), respectively.

PWSF1, PWSF5 and PWSF6 exhibit the composites with the same fineness and inclination angle of spacer yarn, while the thicknesses of these three samples are very close. It can be observed from Figure 14 that the peak force of PWSF1 and PWSF6 are very close and significantly higher than that of PWSF5, indicating that composites with close outer layer structures obtain superior anti-impact properties as compared to the composite with open outer layer structure. The better impact properties of composites PWSF1 and PWSF6 are due to much closer outer layer structures, which can absorb more impact energy. However, the loading stage for PWSF6 is slightly longer than that of PWSF1, indicating that the composite with Locknit on surface layers can absorb more energy than composite made from Chain+Inlay on outer layers, as shown in Figure 15 (b).

PWSF2 and PWSF3 show the composites with the same inclination angle of spacer yarn, but with different spacer yarns' diameters, which are 0.16 and $0.2 \mathrm{~mm}$, respectively. These two specimens also have the similar thickness and outer layer density. Referring to Figure 14 and 15, it can be seen that the composite made from finer spacer yarn obtain higher peak force and longer loading stage. It is demonstrated that composite made from finer spacer yarn endow the superior impact resistance abilities and can absorb more energy, compared to the composite with coarser spacer yarn. The preferable impact performance of composite with finer spacer yarn is due to the higher

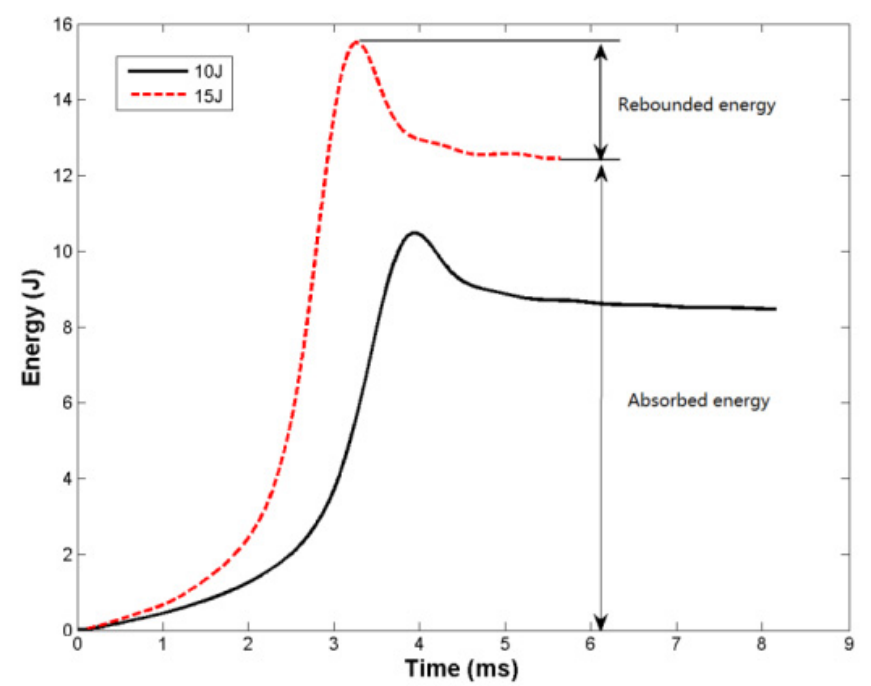

Figure 13. Typical energy-time curve

polyurethane foam volume fraction, which can absorb more energy during impact process.

PWSF1 and PWSF3 represent the composites with different inclination angle of spacer yarn. However, the other parameters of these two samples are very close. Referring to Figure 14, it is evident that the composite with larger inclination angle of spacer yarn exhibits higher peak force, resulting in better antiimpact behaviours for composite made from larger inclination spacer yarn angle. Additionally, the loading stage for composite made from smaller angle of spacer yarn is shorter than that of composite made with larger angle of spacer yarn, which leads 


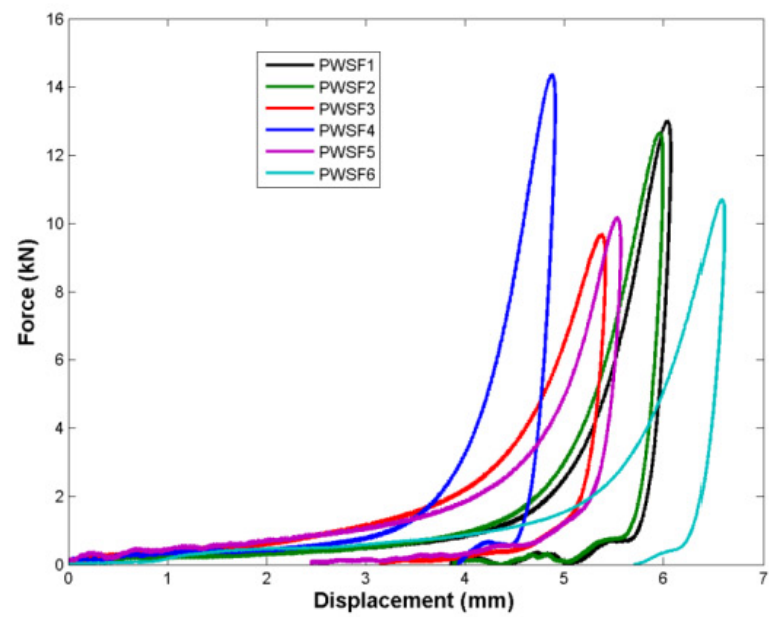

(a) $-10 \mathrm{~J}$

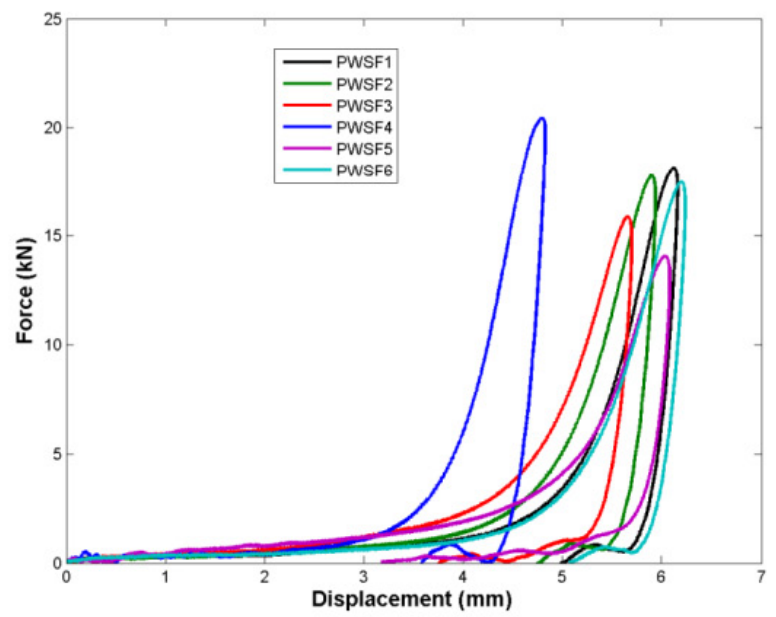

(b) $-15 \mathrm{~J}$

Figure 14. Force-displacement curves for all samples

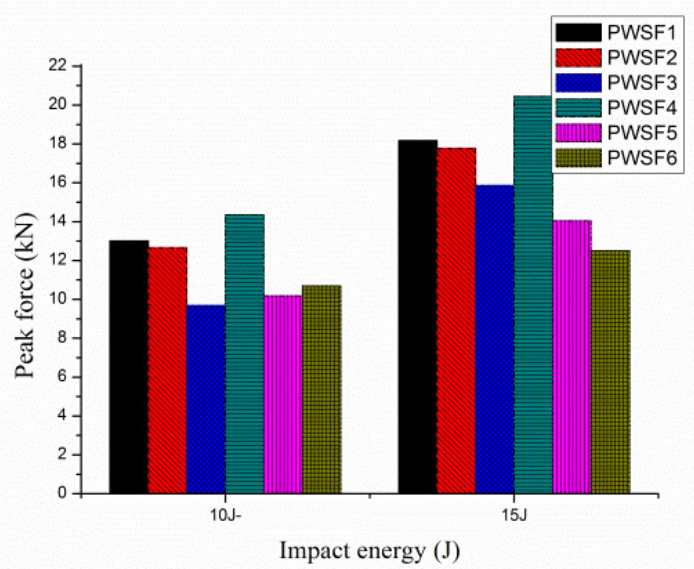

(a)

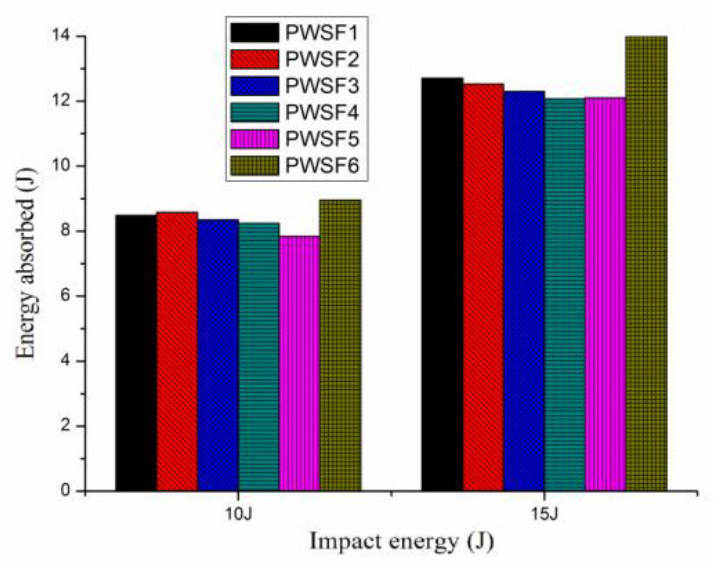

(b)

Figure 15. Peak force and energy absorbed for all samples 
to a decrease in energy absorbed for composite with smaller spacer yarn angle (Figure 15 (b)).

PWSF1 and PWSF4 represent the composites with same outer layer structure and fineness of spacer yarn, but different thickness, which are 8.74 and $6.68 \mathrm{~mm}$. It can be clearly seen from Figure 14 that the thinner composite exhibits higher peak force, but very short loading stage. It might be due to the fact that PWSF4 is too thin to conduct the impact load, implying that the impact load transfers to the support of impact system, instead of absorbing by samples. Obviously, the loading stage for PWSF1 is much longer, compared to that of PWSF4. As a result, the thicker composite endows better energy absorption abilities, which can be seen from Figure 15 (b).

\subsection{Damage of impacting surface of composites}

The damage of impacting surface is an important factor for evaluating the impact resistance properties of composites, since it gives an indication of impact resistance abilities of composites.

The impacting face for PWSF1 tested at $10 \mathrm{~J}$ and $15 \mathrm{~J}$, which is chosen as the representative, is shown in Figure 16. It is evident that the impacting face does not show severe damage. However, in order to effectively evaluate the damage of impacting faces, a XSM-LC 3D scanner instrument was introduced to investigate the impacting surfaces of composites. Figure $17(\mathrm{a})-(\mathrm{b})$ exhibit that impacting surfaces of the same composite (PWSF3) under two different impact energy $(10 \mathrm{~J}$ and $15 \mathrm{~J})$ resemble each other

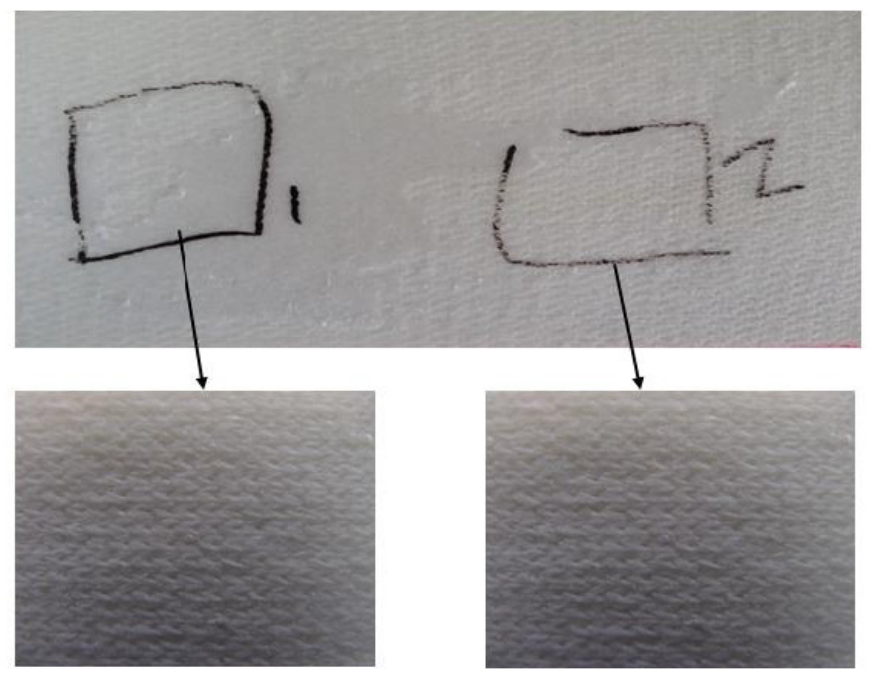

Figure 16. The impacting surface of PWSF1 ('1'represents the position of impact energy of $10 \mathrm{~J}$, and'2'represents the position impact energy of $15 \mathrm{~J})$

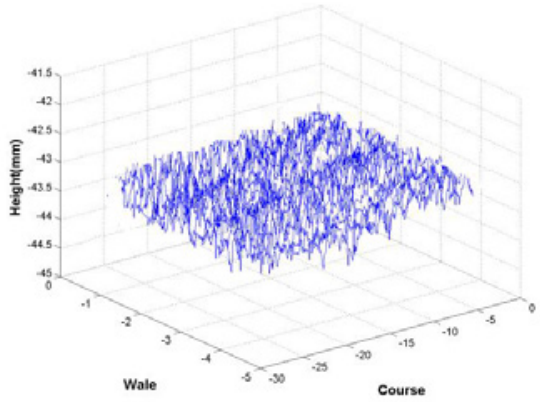

(a) PWSF3-10 J

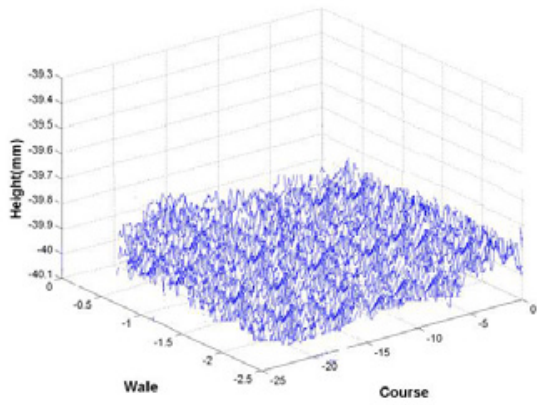

(c) PWSF4-15 J

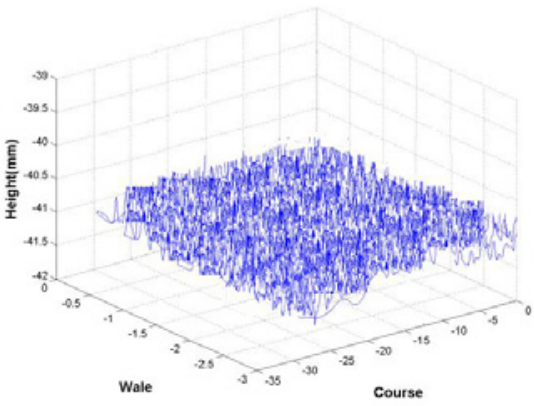

(b) PWSF3-15 J

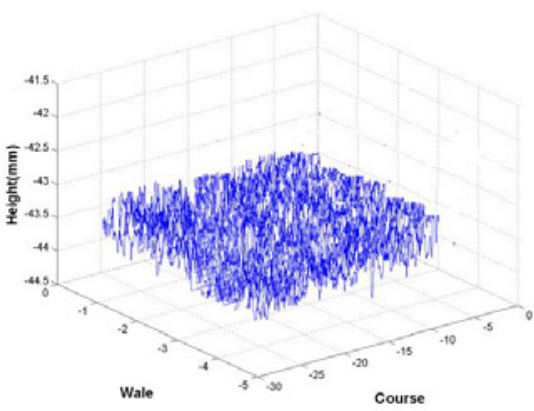

(d) PWSF6-15 J

Figure 17. The impacting surface of composites 
in shape but not in dimension, while the impacting faces of different composites (PWSF4 and PWSF6) with same impact energy (15 J) are shown in Figure 17 (c)-(d). It can be clearly revealed that the height value for these impacting faces is kept in constant levels, indicating that all scanned points on the impacting faces are in the same plane. The findings obtained revealed that the impact energy is effectively absorbed and spread on the impacting faces of composites without damaging the impacting faces. This behaviour is quite different from the previous studies which report that the impacting faces of materials are significantly damaged after impacting. According to the above analysis, it is clear that the composites evaluated in this work possess promising anti-impact and resilience abilities, indicating that the composites are an ideal cushioning materials and its service life can be sustained.

\section{Conclusion}

In the present work, the novel 3D-structure composites with considerably light weight have been successfully manufactured based on 3D warp-knitted spacer fabrics and flexible polyurethane foam. The following conclusions were established through this study:

1. Structural parameters of spacer fabrics have significant influence on the compression and impact properties of 3D-structure composites.

2. The 3D-structure composites are found better with respect to compression resistance capacities when constructed with spacer fabrics having closer surface layer structure and coarser spacer yarn, as well as larger inclination angle of spacer yarn. In contrast, the composites with opener surface layer structure, finer spacer yarn and smaller spacer yarn inclination angle perform worse on the cushioning properties. However, for the composites with different thickness, it is inappropriate to simply compare their compression and energy absorption capacities. In contrast, the allowed stress and deformation must be taken into consideration while selecting the thickness of composites.

3. All the composites investigated in this work perform promising anti-impact and energy-absorbing abilities, indicating that the composites are ideal energy absorber for cushioning applications.

4. The composites obtained in case of spacer fabrics having close outer layer structure, finer spacer yarn, larger spacer yarn inclination angle and higher thickness are found better with respect to impact performance due to superior energy absorption capabilities. In contrast, the impact behaviours of composites with open outer layer structure, coarser spacer yarn, smaller spacer yarn inclination angle and smaller thickness are worse compared to corresponding composites.

Therefore, a conclusion can be drawn that the new type of integrated 3D-structure composites exhibit favourable mechanical performance and their mechanical properties can be tailored to meet the specific end-use requirements by simply varying the fabric structural parameters.

\section{References}

[1] Hahn, M., Otto, A. (2000). Hochtemperature-umforming von zellularemaluminium. Metallschaume, H. P. Degischer (Ed), Wiley-VCH, 432-35.

[2] Velosa, J.C., Rana, S., Fangueiro, R., et al (2011). Mechanical behavior of novel sandwich composite panels based on 3D-knitted spacer fabrics. Journal of Reinforced Plastics and Composites, Vol. 31(2), 95-105.

[3] Liu, Y.P., Hu, H., Zhao, L., et al. (2012). Compression behavior of warp-knitted spacer fabrics for cushioning applications. Textile Research Journal, Vol. 82, 11-20.

[4] Ye, X.H., Hu, H., Feng, X.W. (2008). Development of the warp knitted space fabrics for cushion applications. Journal of Industrial Textiles, Vol. 37, 213-223.

[5] Chen, S., Long, H.R., (2014). Investigation on compression properties of polyurethane-based warp-knitted spacer fabric composites for cushioning applications Part l: experiment. Industria Textila, Vol. 65(4), 200-205.

[6] Abounaim, M., Hoffmann, G., Diestel, O. et al (2011). High performance thermoplastic composite from flat knitted multi-layer textile perform using hybrid yarn. Composite Science and Technology, Vol. 71(4), 511-519.

[7] Abounaim, M., Hoffmann, G., Diestel, O. et al (2009). 3D spacer fabric as sandwich structure by flat knitting for composite using hybrid yarn. AUTEX 2009 World Textile Conference, 26-28 May, 2009, Izmir, Turkey.

[8] Abounaim, M., Hoffmann, G., Diestel, O. et al (2010). Thermoplastic composite from innovative flat knitted3D multi-layer spacer fabric using hybrid yarn and the study of $2 D$ mechanical properties. Composite Science and Technology, Vol. 70(2), 363-370.

[9] Abounaim, M., Hoffmann, G., Diestel, O. et al (2009). Development of flat knitted spacer fabrics for composites using hybrid yarns and investigation of $2 D$ mechanical properties, Textile Research Journal. Vol. 79(7), 596-610.

[10] Vuure, van A.W., Pflug, J., Ivens, J.A., et al (2000). Modeling the core properties of composite panels based in woven sandwich-fabrics performs. Composite Science and Technology, Vol. 60, 1263-1276.

[11] Lin, Y.L., Lu, F.Y., Wang, X.Y. (2006). Experimental study of the compressible behavior of low-density polyurethane foam. Chinese Journal of High Pressure Physics, Vol. 20(1), 89-92.

[12] Miao, X.H., Ge, M.Q. (2009). Indentation force deflection property of cushioning warp-knitted spacer fabrics. Journal of Textile Research, Vol. 30, 43-45.

[13] Mecit, D., Roye, A. (2009). Investigation of a testing method for compression behavior of spacer fabrics designed for concrete applications. Textile Research Journal, Vol. 79(6), 867-87.

[14] Mehment, K., Hakan, Gul, Jan, Ivens et al. (2012). Low velocity impact characteristics of integrated $3 D$ core sandwich composite. Textile Research Journal, Vol. 82(9), 945-962.

[15] Dobrich, O., Gereke, T., Cherif, C. (2014). Modelling of Textile Composite Reinforcement on the Micro-scale. Autex Research Journal, Vol. 14(1),28-33.

[16] Tae, J.K., Cheol, K. (2000). Energy-absorption mechanisms in Kevlar multi-axial warp-knit fabric composites under impact loading. Composite Science and Technology, Vol. 60, 773-784. 